\title{
Research on Adaptive Beamforming of Shape Maintenance Algorithm for the Mainlobe
}

\author{
http://dx.doi.org/10.3991/ijoe.v9iS4.2643 \\ Ji Yan ${ }^{1}$ and Zhang Feng ${ }^{1,2}$ \\ 1 Yulin University, Yulin, China \\ ${ }^{2}$ Northwestern Polytechnical University, Xi'an, China
}

\begin{abstract}
To improve the transmitting adaptive beamforming efficiency and performance of the shape maintenance for the mainlobe, analyzed existing adaptive pattern for beamforming.In this paper, the question that the main lobe of adaptive pattern will be distorted significantly, an approach is proposed to maintain the mainlobe shape based on a main-lobe subspace, and the high peak side-lobe problem is resolved recurring to the orthonormal projection algorithms available. The new approach can be used to the transmitting adaptive beamforming, adaptive sum and sub beam forming. The trace driven simulation shows its performance.
\end{abstract}

Index Terms-adaptive beamforming, shape maintenance, beam, low sidelobe.

\section{INTRODUCTION}

It is often faced with various intentional, unintentional electromagnetic interference of modern radar. It's can greatly enhance the ability of weapon systems, electronic warfare Based on the radar antenna array beamforming, it' $s$ a core technique of modern radar. It is effective for large duty cycle interference adaptive beamforming, because its statistical properties can have the same or similar number of useful data samples to estimate, in such interference direction of the formation of effective interference nulling by adaptive beamforming. As for the similar objective of pulse interference and sidelobe deception target, its existence a short time, multiple samples cannot be used to capture, makes it difficult to adopt adaptive beamforming by adaptive beamforming, usually through low sidelobe level to reduce their impact. As for the tracking radar, the target tracking requires accurate measurement of the location of commonly used single-pulse technique by comparing two or more receive beams (such as beams and difference) to determine the target DOA. To make use of single pulse technique to accurately estimate the target DOA need stable mainlobe shapes. In radar applications, adaptive beamforming adaptive pattern generation must have a low sidelobe level, stable mainlobe shapes, a large duty cycle interference at a deep angle of zero.

These problems can be attributed to find a simple, and the covariance matrix is containing the target signal unrelated, remain static pattern mainlobe (applicable to launch special main valve transmitter and receiver general mainlobe shapes) and sidelobe shape invariant adaptive beam forming method. This paper based on the direction of local conformal thought mainlobe shape preserving and based on the subspace projection sidelobe control joint used for the above problems provide a feasible scheme. The second part of the article describes the direction of local conformal and subspace projection side lobe control method, is proposed based on the fixed shape of the main valve and low sidelobe adaptive beam forming method, and the difference beam and to explain the formation of a separate; The third part of the adaptive from the receiving end and poor adaptive beamforming and launch special mainlobe shape adaptive beam forming two aspects carries on the simulation, verify the effectiveness of the proposed method.

\section{The fiXed Main Lobe Shape And Low Sidelobe ADAPTIVE BEAMFORMING}

SBC conventional adaptive beamforming, such as formula 1:

$$
\min _{\mathbf{w}} \mathbf{W}^{H} \hat{\mathbf{R}} \mathbf{W} \quad \text { s.t. } \quad \mathbf{W}^{H} \mathbf{a}_{q}=1
$$

Where $\hat{\mathbf{R}}$ is the receive data directly or after processing the covariance matrix may contain the target signal $\hat{\mathbf{R}}_{X}$, it may not contain the target signal $\hat{\mathbf{R}}_{I+N}, \mathbf{a}_{q}$ is the normalized static weight vector, that is $\left\|\mathbf{a}_{q}\right\|=1$, For receiving adaptive beamforming is likely to be pointing to the desired direction of steering vector, when the required low sidelobe with Taylor, Chebyshev and amplitude weighting, the receiving end of adaptive and difference beamforming, are static and poor weight vector, and a beam static pattern of low sidelobe through Taylor, Chebyshev weighted implementation etc., Static difference beam pattern can be achieved using Bayesian weighting, and adaptive transmit power for the transmitter vector form, the static weight vector pattern synthesis method may be used to get the right vector, corresponding to a fixed main beam shape and sidelobe envelope .

Formula 1 of the data covariance matrix in the presence of the target signal for adaptive pattern of impact is enormous, measuring adaptive beamforming algorithm one of the criteria of a good or bad is to see there is no interference adaptive weight vector is equal to static power, of course, equal to the static power is what we hope, corresponds to formula 1 when the covariance matrix containing the target signal and the static weight vector is not equal to the desired signal steering vector, the adaptive interference does not exist right does not mean static weight vector, a serious distortion of the main lobe appears, shown in Figure 1: Adaptive finite snapshots 


\section{SPECIAL FOCUS PAPER

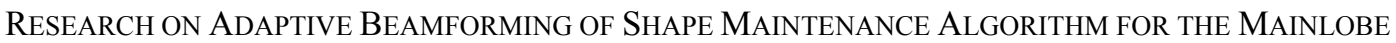

difficult to maintain the original static pattern of the sidelobe, the main lobe of emission for a particular adaptive pattern, main lobe was also affected, when the adaptive weight vector used to generate the data containing the target signal adaptive is to cause the formation of nulling in the direction of the target (when the static weight vector and the true direction of the target is not the same, but in practice is usually the case, Figure 1 for the 22 half-wavelength array element uniform linear.

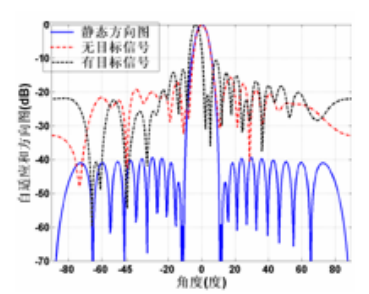

Figure 1a. Sum beam pattern

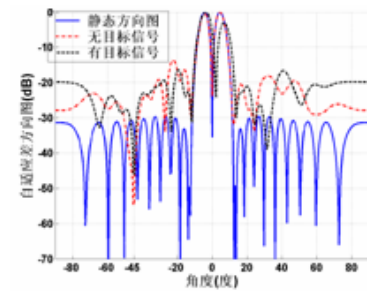

Figure 1b. Sub beam pattern

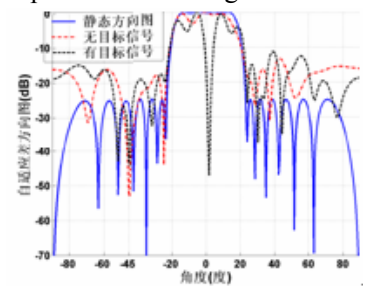

Figure 1c. Emission flat pattern

\section{A. The existing beam-forming method}

The existing beam-forming method is to add a static weight vector increment, that is $\mathbf{w}=\mathbf{a}_{q}+\Delta \mathbf{w}$, to meet the suppression of interference as small as possible while making incremental, which can be a multi-objective optimization problem representation:

$$
\left\{\begin{array}{l}
\min _{\Delta \mathbf{w}} \Delta \mathbf{w}^{H} \Delta \mathbf{w} \\
\min _{\Delta \mathbf{w}}\left(\mathbf{a}_{q}+\Delta \mathbf{w}\right)^{H} \hat{\mathbf{R}}\left(\mathbf{a}_{q}+\Delta \mathbf{w}\right)
\end{array}\right.
$$

For the formula 2 does not actually easy operation, besides the general purpose through the design of weight multi-objective problem into single objective optimization problem, in practice often use two simplified deformation:

$$
\min _{\Delta \mathbf{w}}\|\Delta \mathbf{w}\| \quad \text { s.t. } \quad\left(\mathbf{a}_{q}+\Delta \mathbf{w}\right)^{H} \mathbf{C}=\mathbf{f}
$$

and

$$
\min _{\Delta \mathbf{w}}\left(\mathbf{a}_{q}+\Delta \mathbf{w}\right)^{H} \hat{\mathbf{R}}\left(\mathbf{a}_{q}+\Delta \mathbf{w}\right) \quad \text { s.t. }\|\Delta \mathbf{w}\| \leq \xi
$$

Where $\mathbf{C}$ is the constraint matrix, $\mathbf{f}$ is the vector of constraint values. that by constraint to achieve disturbance suppression Formula 3 , so need to predict the interference direction vectors or interference subspace, and formula 4 passes to the incremental modulus constraint to achieve the ultimate direction of static direction map remained, but the interference nulling is not easy to predict the impact caused, and selection of $\xi$ is an experience value.

\section{B. The fixed main lobe shape and low sidelobe adaptive beamforming}

1) adaptive realization of the fixed shape of the main lobe

The weight vector changes but to ensure that the same main lobe shape, the sufficient condition is the main lobe of the right to decide the shape vector in the weight change, according to the main valve main valve subspace estimation: (assuming the array number for $M, \Theta_{m}$ flap angle range )

$$
\mathbf{R}_{\Theta_{m}}=\int_{\theta \in \Theta_{m}} \mathbf{a}(\theta) \mathbf{a}(\theta)^{H} d \theta \stackrel{E V D}{=} \sum_{i=1}^{M} \lambda_{i} \mathbf{v}_{i} \mathbf{v}_{i}^{H}, \quad \lambda_{1} \geq \lambda_{2} \geq \mathrm{L} \quad \lambda_{M}
$$

The main lobe steering vector sum of points obtained by sampling the angle in formula 5 , set $\lambda_{1} \geq \lambda_{2} \geq \mathrm{L} \geq \lambda_{M}$, ataking the value of the formula 5 major feature of the composition corresponding to the main lobe of sub-feature vector space, take a large number of eigenvalues of $P$ power distribution can be determined:

$$
P=\min _{k}\left\{\frac{\sum_{i=1}^{k} \lambda_{i}}{\sum_{i=1}^{M} \lambda_{i}} \geq \gamma\right\}
$$

The main lobe and its sub-space a set of orthogonal sub-space form the base of the column space of matrix and the corresponding projection matrix are:

$$
\mathbf{U}_{m}=\left(\mathbf{v}_{1}, \mathbf{v}_{2}, \mathrm{~L}, \mathbf{v}_{P}\right), \mathbf{U}_{m}{ }^{\perp}=\left(\mathbf{v}_{P+1}, \mathbf{v}_{P+2}, \mathrm{~L}, \mathbf{v}_{M}\right)
$$

$$
\mathbf{P}_{m}=\mathbf{U}_{m}\left(\mathbf{U}_{m}{ }^{H} \mathbf{U}_{m}\right)^{-1} \mathbf{U}_{m}{ }^{H}, \mathbf{P}_{m}{ }^{\perp}=\mathbf{I}-\mathbf{P}_{m}
$$

The entire $M$ space can be divided into the main valve subspace and its orthogonal subspace, the corresponding weight vector can be written as:

$$
\mathbf{W}=\mathbf{W}_{m}+\mathbf{W}_{m}{ }^{\perp}=\mathbf{P}_{m} \mathbf{W}+\mathbf{P}_{m}{ }^{\perp} \mathbf{W}
$$

So when $\mathbf{W}$ changes its weight $\mathbf{W}_{m} \equiv \mathbf{W}_{m 0}$ $\mathbf{P}_{m} \mathbf{a}_{q}$ unchanged, then the main lobe corresponding to the same pattern, as opposed to formula 1, the main lobe conformal adaptive pattern generation:

$$
\min _{\Delta \mathbf{W}}\left(\mathbf{W}_{n 0}+\mathbf{U}_{m}{ }^{\perp} \Delta \mathbf{W}\right)^{H} \hat{R}\left(\mathbf{W}_{n 0}+\mathbf{U}_{m}^{\perp} \Delta \mathbf{W}\right) \text { s.t. }\left(\mathbf{W}_{n 0}+\mathbf{U}_{m}{ }^{\perp} \Delta \mathbf{W}\right)^{H} \mathbf{a}_{q}=1
$$

The corresponding solution can be expressed as:

$$
\Delta \mathbf{W}=\left(\mathbf{U}_{m}{ }^{\perp H} \hat{\mathbf{R}} \mathbf{U}_{m}{ }^{\perp}\right)^{-1} \mathbf{U}_{m}{ }^{\perp H}\left(\hat{\mathbf{R}} \mathbf{W}_{m 0}-\lambda \mathbf{a}_{q}\right)
$$




$$
\lambda=\frac{1-\mathbf{W}_{m 0}{ }^{H} \mathbf{a}_{q}+\mathbf{W}_{m 0}{ }^{H} \hat{\mathbf{R}} \mathbf{U}_{m}{ }^{\perp}\left(\mathbf{U}_{m}{ }^{\perp H} \hat{\mathbf{R}} \mathbf{U}_{m}{ }^{\perp}\right)^{-1} \mathbf{U}_{m}{ }^{H H} \mathbf{a}_{q}}{\mathbf{a}_{q}{ }^{H} \mathbf{U}_{m}{ }^{\perp}\left(\mathbf{U}_{m}{ }^{\perp H} \hat{\mathbf{R}} \mathbf{U}_{m}{ }^{\perp}\right)^{-1} \mathbf{U}_{m}{ }^{\perp H} \mathbf{a}_{q}}
$$

From the perspective of constraint, formula (10a) is equivalent to:

$$
\min _{\mathbf{W}} \mathbf{W}^{H} \hat{\mathbf{R}} \mathbf{W} \quad \text { s.t. } \quad \mathbf{C}^{H} \mathbf{W}=\mathbf{f}
$$

$$
\text { Where } \mathbf{C}=\left(\mathbf{a}_{q}, \mathbf{U}_{m}\right), \mathbf{f}=\left(\begin{array}{c}
1 \\
\mathbf{U}_{m}{ }^{H} \mathbf{a}_{q}
\end{array}\right) \text {, Resulting in: }
$$

$$
\mathbf{W}=\hat{\mathbf{R}}^{-1} \mathbf{C}\left(\mathbf{C}^{H} \hat{\mathbf{R}}^{-1} \mathbf{C}\right)^{-1} \mathbf{f}
$$

It can verify, when $\hat{\mathbf{R}}=\sigma_{n}^{2} \mathbf{I}$ or $\hat{\mathbf{R}}=\sigma_{0}^{2} \mathbf{a}\left(\theta_{0}\right) \mathbf{a}\left(\theta_{0}\right)^{H}+\sigma_{n}^{2} \mathbf{I}$, that is, without interference $\mathbf{W}=\mathbf{a}_{q}$, Where $\sigma_{0}^{2}$ and $\sigma_{n}^{2}$ are the target signal and noise power, ${ }^{\mathbf{a}\left(\theta_{0}\right)}$ as the goal of true steering vector.

Note that in solving (10b) when the assumption is not entirely static weight vector subspace in the main lobe, the pattern synthesis or the main beam pointing direction vector amplitude weighting results. And when a located entirely within the main valve subspace (corresponding to the static weight vector without low sidelobe), then directly to remove the constraint (10a) model can be solved (because the adaptive weight vector $\left(\mathbf{P}_{m} \mathbf{a}_{q}+\mathbf{U}_{m}{ }^{\perp} \Delta \mathbf{W}\right)^{H} \mathbf{a}_{q}=\mathbf{a}_{q}{ }^{H} \mathbf{P}_{m} \mathbf{a}_{q} \equiv$ Const $)$.

\section{2) Realization of adaptive low side lobe}

As in the introduction pointed out how the adaptive get low sidelobe is a wide range of research questions, including aspects of study is in static pattern to ensure low sidelobe circumstances how to design adaptive method. Many studies have analyzed the adaptive pattern sidelobe level distortion reason: Finite snapshots divergence caused by the noise eigenvalue eigenvector in the generation of adaptive weight vector, thus eliminating the noise adaptive weight vector in the feature vector components (which can be attributed to the projection method) has become an easy to maintain the adaptive original static pattern method of low sidelobe characteristics, whether the data covariance matrix containing the target signal, the typical projection algorithm has the following two:

$$
\begin{gathered}
\mathbf{W}^{\prime}=\mathbf{P}_{n} \mathbf{a}_{q} \\
\mathbf{W}^{\prime}=\mathbf{P}_{s} \mathbf{W}_{a d 0}
\end{gathered}
$$

(12a) in $\mathbf{a}_{q}$ is the static weight vector, that is bound vector, the orthogonal projection algorithm applies only to data in the case of non-target signal for interference when the strong adaptive weight vector of the ideal approximation, by the time the signal subspace interference Zhang, after the projection direction to ensure the formation of the interference nulling for adaptive pattern specially designed sidelobe characteristics (if any) does not necessarily cause damage; (12b) applied to the data containing the target signals, $\mathbf{W}_{a d 0}$ as the conventional adaptive methods (such as LCMV) to get the adaptive weight vector, the $\mathbf{W}_{\text {ado }}=\mu \hat{\mathbf{R}}^{-1} \mathbf{a}_{q}$ is the static weight vector in the signal subspace, ideal adaptive weight vector in the signal subspace, when the static weight vector is specifically designed to meet a certain main lobe or sidelobe envelope shape, (12b) type simple projection will destroy this performance. As for the noise or signal subspace by different methods.

The adaptive weight vector as the static weight vector with an adaptive incremental, as shown in formula (13):

$$
\mathbf{W}_{a d 0}=\mu \mathbf{R}^{-1} \mathbf{a}_{q}=\mathbf{W}_{a d 0}=\frac{\mu}{\sigma_{n}^{2}}\left(\mathbf{a}_{q}-\sum_{i=1}^{P} \frac{\lambda_{1}-\sigma_{n}^{2}}{\lambda_{1}}\left(\mathbf{v}_{i}^{H} \mathbf{a}_{q}\right) \mathbf{v}_{i}\right)=\frac{\mu}{\sigma_{n}^{2}}\left(\mathbf{a}_{q}-\mathbf{V x}\right)
$$

The $\mathrm{V}$ column for the signal subspace of a group of radicals. From another perspective, ideally adaptive weight vector in the static weight vector on the variation of signal subspace of a group is a linear combination, and then the unconstrained projection algorithm can be more generally written as:

$$
\mathbf{W}^{\prime}=\frac{\mu}{\sigma_{n}^{2}} \mathbf{a}_{q}+\mathbf{P}_{s}\left(\mathbf{W}_{a d 0}-\frac{\mu}{\sigma_{n}^{2}} \mathbf{a}_{q}\right)
$$

Formula (4) is not affected by the static weight vector performance and data not found in the target signal limiting.

At the same time easy to prove, the formula (3) of the beam-forming, the adaptive weight:

$$
\mathbf{W}=\mathbf{a}_{q}-\mathbf{C}\left(\mathbf{C}^{H} \mathbf{C}\right)^{-1} \mathbf{C}^{H} \mathbf{a}_{q}=\left(\mathbf{I}-\mathbf{C}\left(\mathbf{C}^{H} \mathbf{C}\right)^{-1} \mathbf{C}^{H}\right) \mathbf{a}_{q}
$$

C is the interference steering vector or their sheets into the space, then the $\left(\mathbf{I}-\mathbf{C}\left(\mathbf{C}^{H} \mathbf{C}\right)^{-1} \mathbf{C}^{H}\right)$ on the noise subspace, and (12a) the same result, but also the equivalent of Sidelobe cancellation (static weight vector channel as the main beam channel to interfere with the normal direction beamforming auxiliary channel beam) results.

3) the fixed main lobe shape and low sidelobe adaptive beamforming

The first step: conformal adaptive main lobe, corresponding to formula (10), the need to determine where the main lobe of conformal region, and then calculate the main lobe subspace. Been the main lobe conformal adaptive weight vector $\mathbf{W}_{a d 0}$. Step two: The first step produces the weight vector corresponding to eliminate the effects of random noise feature vector, corresponding to the formula (12b) operation to get the final adaptive weight vector $\mathbf{W}^{\prime}$.

For the mainlobe can be considered sub-space calculated in advance and save, for the receiver to beam scanning, they may also consider whether to seek a simple iterative algorithm: from a starting point to the main lobe subspace by a simple process to get the next point to point on the main lobe subspace. 


\section{RESEARCH On AdAPtive BEAMForming OF SHAPE MAINTENANCE AlgORITHM FOR THE MAINLOBE}

\section{Simulation AND PERFORMANCE ANALYSIS}

In the 22 array element half wavelength of uniform linear array as an example, figure 1 three simulation results for this array simulation results. The presence of both target signal, namely, the direction of the main lobe with the circumstance of the target.

Simulation 1: comparison with conventional adaptive beamforming

Use this method to get the corresponding diagram in Figure 1 three results, in order to compare the results, the results of the conventional adaptive once again in the graph. Target signal is $20 \mathrm{~dB}$, the direction of $2^{\circ},-45^{\circ}$ there is $40 \mathrm{~dB}$ interference direction, taking snapshots 256 .
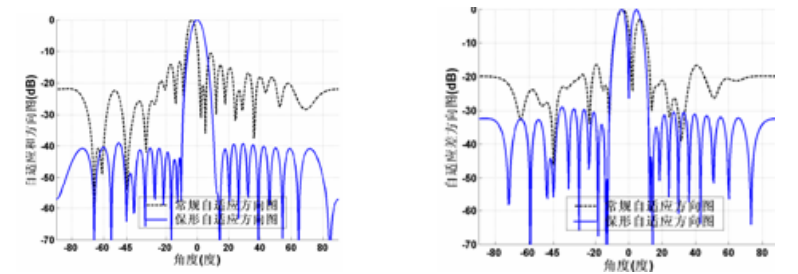

Figure 2a. Adaptive sum beam pattern Figure 2b. Adaptive sub beam pattern

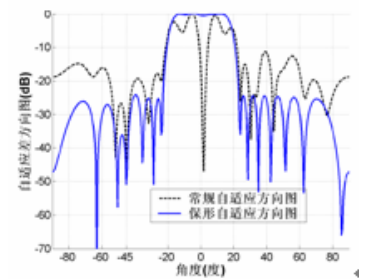

Figure 2c. Adaptive transmitter flat pattern

Compared with conventional adaptive methods can be seen, this approach not only in the mainlobe and sidelobe envelope pattern to maintain a static shape in the direction of the formation of the zero-interference should be deeper than the conventional adaptive trap method, because the relative conventional adaptive in terms of its direction does not require the formation of the target nulling.

Simulation 2: with the existing method is adaptive beam conformal

Compared to simulation 1 , the number increased interference in $-45^{\circ},-30^{\circ}, 40^{\circ} 40 \mathrm{~dB}$ increase in the direction of interference in the interference direction is assumed to use (3) corresponds to the conformal method, for comparison, corresponds to Figure 3.
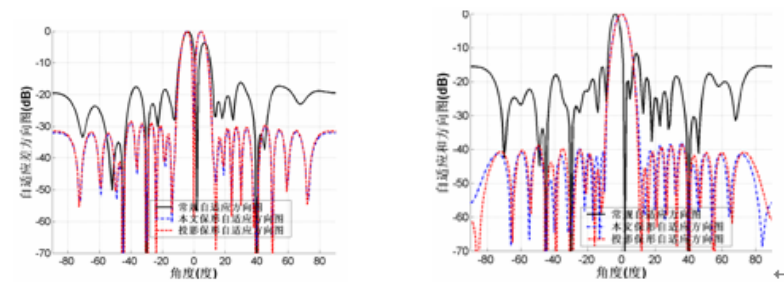

Figure 3a. Adaptive sum beam pattern Figure 3b. Adaptive sub beam pattern

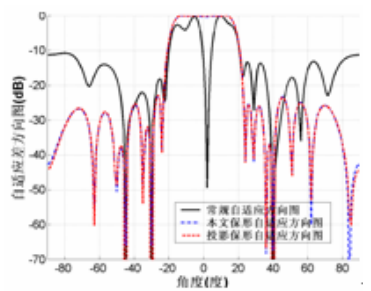

Figure 3c. Adaptive transmitter flat pattern
From the above results can be out in the interference far away from the main lobe, the present method and interference under the direction of the known formula (3) of the conformal method performs as well, but interference from the main valve close, or the presence of interference in the main valve, the method of this paper to save the main lobe shape precedence interference suppression, and the formula (3) corresponding to the conformal method is interference suppression takes precedence over saving the main lobe shape, which is the first side lobe suppression using conventional methods and then use other methods to interfere with eliminate the main lobe interference in terms of the method provides a sidelobe suppression under main lobe interference conformal method.

Simulation 3: The main lobe subspace estimate the selection of the main lobe in the conformal properties

Increase in the previous simulation, based on a $10^{\circ}$ direction $40 \mathrm{~dB}$ interference, where only the flat-topped emission pattern simulation, Figure $4 \mathrm{a}, 4 \mathrm{~b}, 4 \mathrm{c}$ are $\gamma=$ $0.9999, \gamma=0.999999, \gamma=0.99999$, and the result shows that the closer the selected $\gamma 1$, the main valve to maintain the better, because this time the main valve subspace has more dimensions, while the main lobe of the orthogonal subspace of the vector affect the shape of the main lobe is smaller, but the direction of the formation of the interference nulling depth will be reduced.

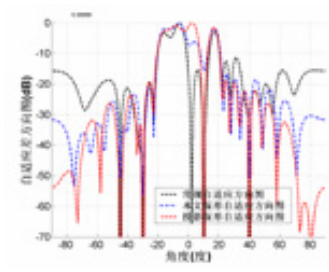

Figure 4a. $\gamma=0.9999$

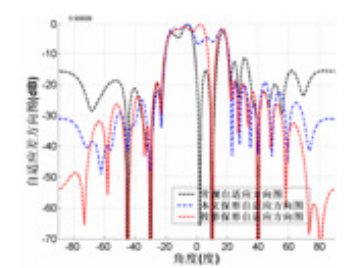

Figure 4b. $\gamma=0.99999$

\section{Performance Tests}

In this paper, a summary and comparison of the existing adaptive beamforming pattern control method was proposed based on the main valve, conformal and sidelobe envelope conformal adaptive beam forming method, solves the following three problems: the static weight vector using a specially designed ( differs from the target direction vector ) containing difference of low sidelobe forming problems, because adaptive and difference beamforming can use the data must contain the target signal; arbitrary static firing pattern adaptive nulling problem.

\section{ACKNOWLEDGMENT}

This work is partially supported by the Science and Technology Research and Development Program of Shaanxi Province in 2012 \#2012K12-03-08, Funding Project for Department of Education of Shaanxi Province in 2012 \#12JK0537. Thanks for the help.

\section{REFERENCES}

[1] Yu HJ, Liu YQ, Zhang M, Ru LY, Ma SP. Research in search engine user behavior based on log analysis. Journal of Chinese Information Processing, 2007, 21(1):109-114 (in Chinese with English abstract).

[2] Liu YQ, Zhang M, Ru LY, Ma SP. Automatic query type identification based on click through information. In: $\mathrm{Ng} \mathrm{HT}$, 
Leong MK, Kan MY, Ji DH, eds. Proc. of the 3rd Asia Information Retrieval Symp., AIRS 2006. LNCS 4182, Berlin, Heidelberg: Springer-Verlag, 2006. 593-600.

[3] Hosseini, S. , Cox, J. R. Optimal solution of off-line and on-line generalized caching. Technical Report, WUCS-96-20, washington University at St. Louis, 1996.

[4] [1] G.M. Herbert, Malvern, Low sidelobe pattern synthesis and subspace projection, IEEE,2006.

[5] [2] G.M. Herbert, A new projection based algorithm for low sidelobe pattern synthesis in adaptive arrays, IEE Radar '97 conference proceeding, IEE pub. No.449, pp. 396-400, October 1997.

[6] [3] Renbiao Wu, Zhisong Wang, Dan Lu etc, Further results on peak sidelobe control in adaptive arrays, IEEE,2006.

[7] [4] Kai-Bor Yu, David J.Murrow, Adaptive digital beamforming for angle estimation in jamming, IEEE,2001.

[8] [5] Sung-Hoon Moon, Dong-Seog Han, Hae-Sock Oh etc, Monopulse angle estimation with constrained adaptive beamforming using simple mainlobe maintenance technique, IEEE, 2003.

\section{AUTHORS}

Ji Yan received the MS degree in Computer science from Xidian University in 2008. Now he is an associate professor at the Institute of energy engineering in Yulin University. His research interests are in the areas of modeling of complex systems, the Internet of Things applications. (e-mail: donguncn@126.com).

Zhang Feng received the MS degree in Computer science from Xidian University in 2009. Now he is a PhD of Northwestern Polytechnical University. He is currently a associate professor at the School of automation, in Yulin University. His research interests are in the areas of Cloud integrated manufacturing technology, the modeling of complex systems, the Internet of Things applications. (email: tfnew21@sina.com).

This work was supported in part by Shaanxi Province in 2012 \#2012K12-03-08. It is an extended and modified version of a paper presented at the International Conference on Mechanical Engineering, Automation and Material Science (MEAMS2012), held 22-23 December 2012, Wuhan, China. Received 17 January, 2013. Published as resubmitted by the authors on 16 May, 2013. 